\title{
Analysis of Arid Area Urban Water Resources Bearing Capacity Based on NARSD Model
}

\author{
Xuanchang Gao \\ North China Electric Power University, Baoding, Hebei province, China \\ Email:1571264661@qq.com
}

Keywords: SD model ANN model Water Scarcity Bearing Capacity

\begin{abstract}
Water resources shortage is a problem paid general attention to in today's world. How to predict the clean water provided in a region become universal concern ever since. So we develop a NARSD Model to analysis water bearing capacity with the rate of water supply to water demand. It is improved from the traditional SDM (System Dynamics Model) which takes the correlations of variables in a system into consideration but is inflexible in parameters determination. So the feedback of the NARM is used to get main parameters, which get over the disadvantages of the $S D M$. Many factors that affect the water resources of quantity and quality are taken into account, for instance, water for domestic, industry, agriculture et al. In order to demonstrate the effectiveness and feasibility of the above methods, We choose Beijing, an over-exploited city, to analyze how and why the water is scarce from the view of physical scarcity and economical scarcity and water resources bearing capacity. Finally, effective intervention will be put forward to solve relevant issues.
\end{abstract}

\section{Introduction:}

The growing scarcity of fresh and clean water is among the most important issues facing civilization in the $21^{\text {st }}$ century. Therefore, a reasonable analysis and effective intervention are strongly demanded to alleviate and ultimately solve the water scarcity, which will also be the main concern in this paper. We measure the water scarcity from the view of water demand and water supply with the rate of water supply to water demand. Then we set up a Neural Network Model to measure the water scarcity of Beijing, considering that the ANN model hasn't taken the inner correlation among different parameters. So we then build up a System Dynamic Model(SD) that takes the inner correlation into account. However, the result of it has still a relative error, the reason of which may be its inflexibility in parameters determination. So we set up an improved model - the NARSD Model which is the combination of the NAR Model and the traditional SD Model.

\section{The basic assumptions:}

Clean water here cannot be influenced by the waste water produced from different area.

\section{Method:}

-Water scarcity in view of water demand and water supply

For analysis of the ability of a region to provide clean water to meet the needs of its population, that is, to assess the water scarcity in this region, an evaluation standard should be set up in the first place. Water scarcity can be described with water demand and water supply.

Hence, the extent of water scarcity can be described with the rate $\alpha$ :

$$
\alpha=\frac{d}{u} \times 100 \%
$$

Where: $d$ is the amount of total water demand, $u$ is the amount of total water supply.

Meanwhile, the water demand can be mainly divided into four parts: Domestic water consumption, Water consumption for Environment, Water consumption for Industry and Water consumption for Primary Industry. However, the water supply is composed mainly with five main parts: Precipitation utilized quantity, Entry water, Reclaimed water, Exit water and Sea water 
desalination as is shown in Fig. 1.

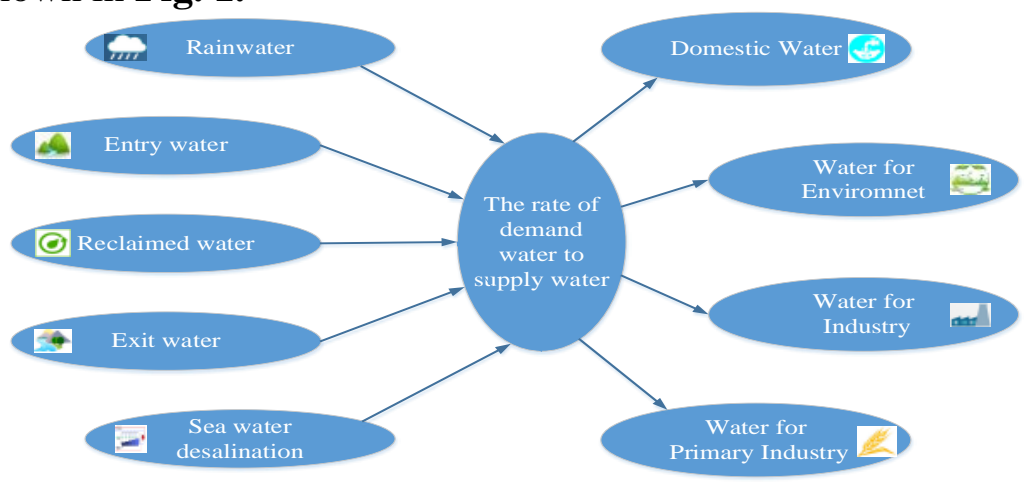

- ANN model:

Fig. 1 The composition for water demand and water supply

Based on the statistic from 2003 to 2014 of water resources in Beijing, we use the Neural Network Model to estimate the water demand, water supply as well as the rate $\alpha$, all of them are then compared with the actual value, and the results are shown in Table 1.

Table 1. The comparison between actual value and predicted value by the Neural Network Model

Units:0.1 billion cubic meters

\begin{tabular}{cccc}
\hline Value & $d$ & $u$ & $\alpha$ \\
\hline Actual Value & 37.49 & 20.30 & $54.15 \%$ \\
Predicted Value & 35.41 & 16.29 & $46.01 \%$ \\
Relative Error & $5.55 \%$ & $19.75 \%$ & $15.03 \%$ \\
\hline
\end{tabular}

From the results listed in the table above, ANNM can be eventually used to measure water scarcity. However, it only takes the primary elements separately into account without considering the social and economic issues and the correlations among them.

-The Basic System Dynamic Model (SDM)

A System Dynamic Model (SDM) can not only arrange and describe the complicated connections among each element in different levels, but also deal with dynamic processes with feedback in a system. The approach of SDM is decomposing the system into layers, that is, dividing the system into several sub-systems, description of sub-systems is then established. The steps can be described as follows:

1)Decomposed the system into several sub-systems that are inter connected according to the structure and layer of the system.

2) Describe the sub-systems.

$$
\dot{L}=P R \quad\left[\begin{array}{l}
R \\
A
\end{array}\right]=W\left[\begin{array}{l}
L \\
A
\end{array}\right]
$$

The variable in SDM can mainly be divided into state variable $(L)$, rate variable $(R)$, auxiliary variable $(A)$, exogenous variable $(E)$ and constant $(C)$.In addition to $(C)$ and $(E)$, the development and changes of other variables are derived from the results of internal and external feedback. The above-described mechanism can be shown in Fig. 2

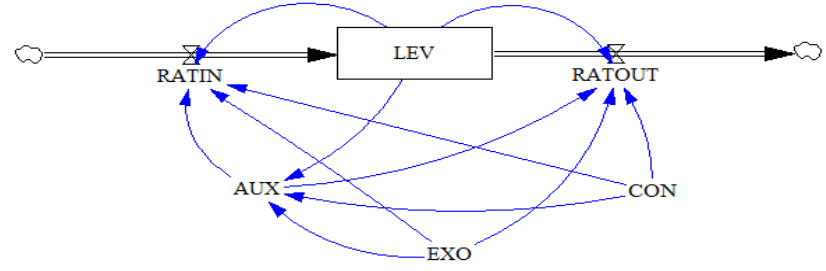

Fig. 2 The variable flow diagram

-Application of the SD Model

The SD Model, which takes the dynamic correlation among different elements into account, does well in regarding social and economic issues as a whole system for analysis of water scarcity. Hence, we apply the SD model to assess the water demand and water supply to analyze the water scarcity. There we also take Beijing for example.The flow diagram is showed in Fig. 3 and Fig. 4 
which are drawn based on the causality diagram above.

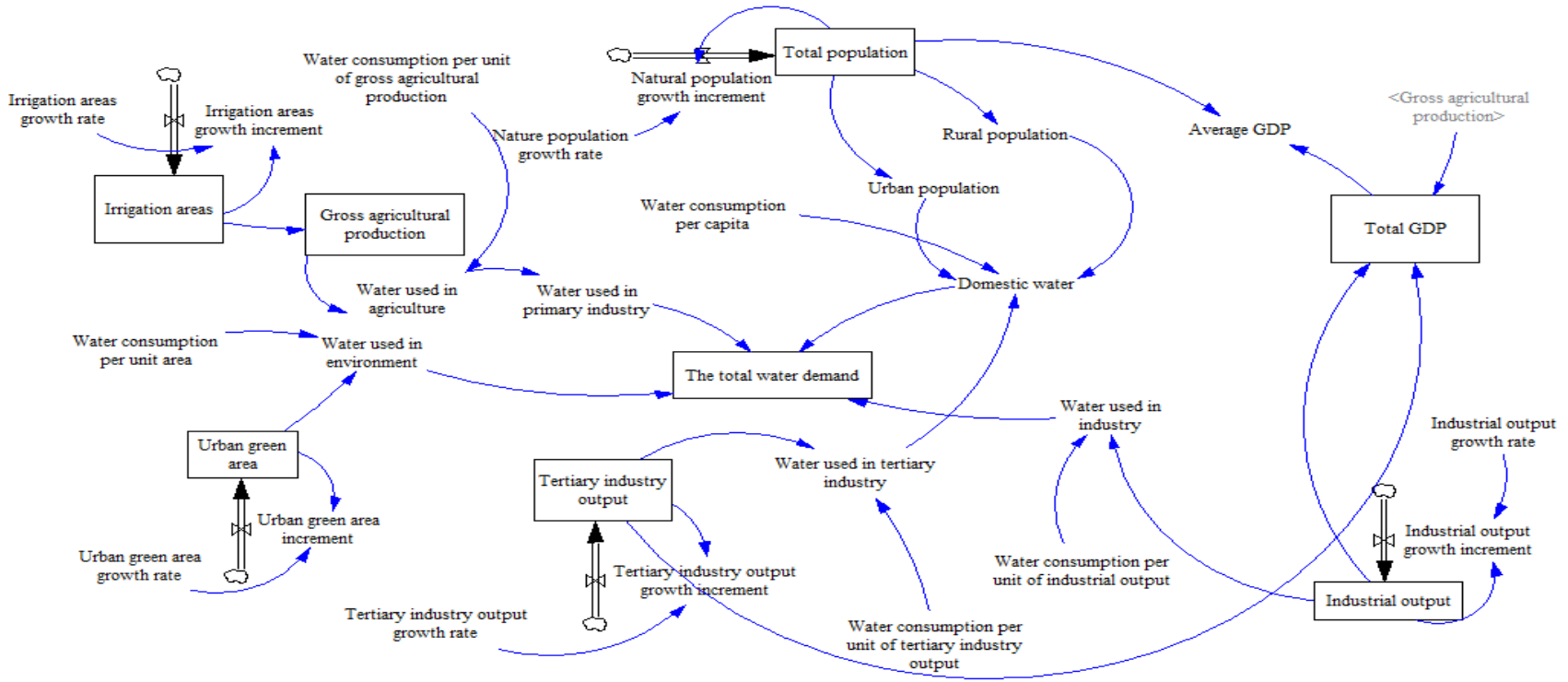

Fig. 3 The flow graph of water demand simulated with the Vensim software

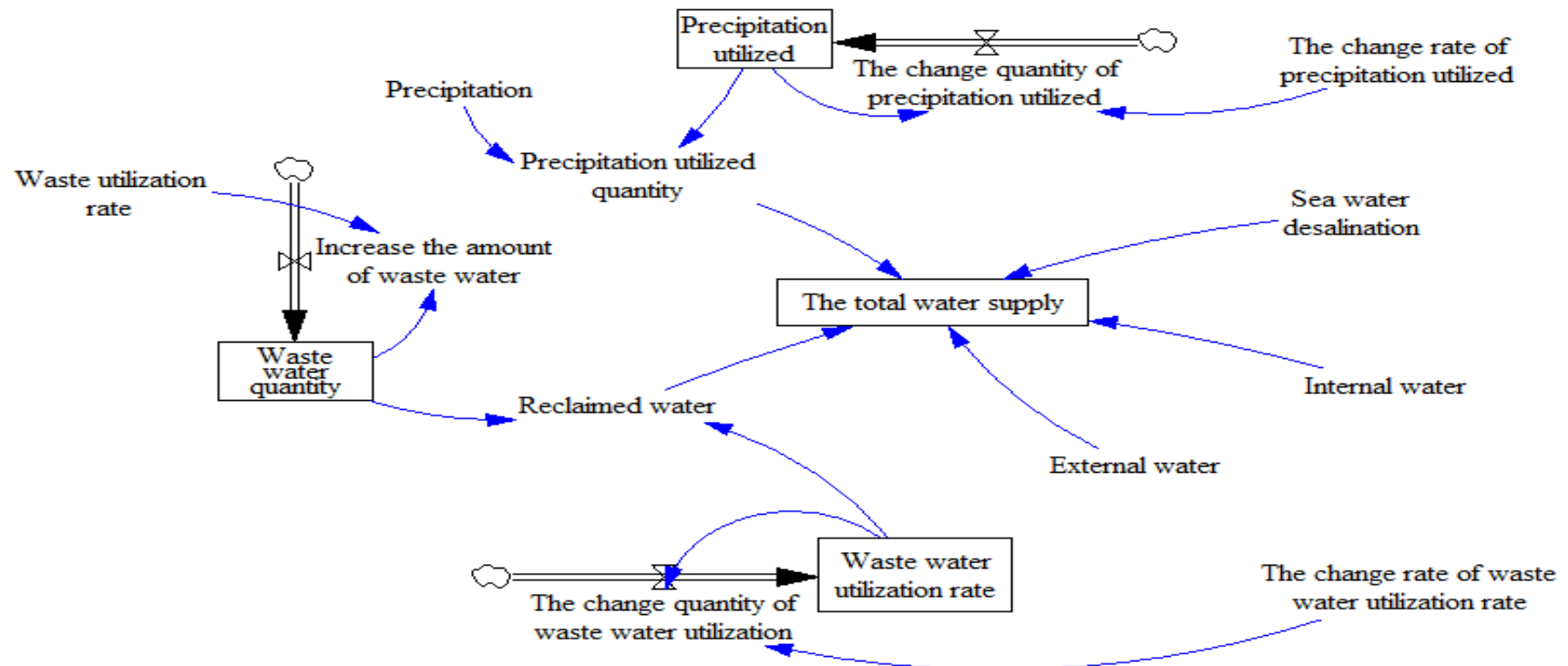

Fig. 4 The flow graph of water supply simulated with the Vensim software

The results are as follows:

Table 2.The comparison between actual value and predicted value with the SD Model in 2014 Units: 0.1 billion cubic meters

\begin{tabular}{cccc}
\hline Value & $d$ & $s$ & $\alpha$ \\
\hline Actual Value & 37.49 & 20.30 & $54.15 \%$ \\
Predicted Value & 36.20 & 22.50 & $62.15 \%$ \\
Relative Error & $3.44 \%$ & $10.83 \%$ & $14.78 \%$ \\
\hline
\end{tabular}

As we can learn from this table, there is still a deviation in the results, which is lower than that in the Neural Network, but still may be ascribed to the parameters determination. The reason of the deviation may lie in its flexible in parameters determination, that is, the main parameters will keep unchanged once determined. So we consider developing a new model which can get over this advantage.

-The Improved System Dynamic Model (NARSDM)

NAR (Nonlinear auto-regressive) Model is special form of NARMA (Nonlinear auto-regressive Moving Average) Model. When the data is time series, and the input of model is unknown, the expression can be rewritten as follows in a ANR form:

$$
y_{n}=F\left(y_{n-1}, \ldots, y_{n-k}\right)+k \varepsilon_{n}
$$


Where: $F$ is the nonlinear function; $k$ is the constant, $\varepsilon_{n}$ is the random variable subject to Gauss distribution.

The output of each $y$ in the NAR model is to point to the input of the neural network layer, serving as the next output of the adjustment parameters and completing the adjustment of the neural network. Therefore, the NAR model can reflect the historical information of the system, and it can be used to parameters determination in the SD Model. Hence, the NARSD Model is the combination of the NAR Model used to determine the main parameters and the SD Model used to analyze the inner correlations, which is believed to perform better in the parameters determination.

Result:

Table 3.The Actual Value and Predicted Value with the NARSDM in 2014 Units: 0.1 billion cubic meters

\begin{tabular}{cccc}
\hline Value & $d$ & $s$ & $\alpha$ \\
\hline Actual Value & 37.49 & 20.30 & $54.15 \%$ \\
Predicted Value & 37.22 & 22.24 & $59.75 \%$ \\
Relative Error & $0.72 \%$ & $9.50 \%$ & $10.34 \%$ \\
\hline
\end{tabular}

The results of the Neural Network Model, the basic SD Model and the improved NARSD Model are listed in the Table 4. for comparison.

Table 4. The relative error of the three models

\begin{tabular}{cccc}
\hline Value & $d$ & $s$ & $\alpha$ \\
\hline NN Model & $5.55 \%$ & $19.75 \%$ & $15.03 \%$ \\
SD Model & $3.44 \%$ & $10.83 \%$ & $14.78 \%$ \\
NARSD Model & $0.72 \%$ & $9.50 \%$ & $10.34 \%$ \\
\hline
\end{tabular}

\section{Summary:}

In conclusion, The NARSD Model combines the systematicness of SD Model and the autoregression of NAR Model, making the main parameters of the SD Model renewed by NAR Model and every quantity in system contacted by SDM. So we can use NARSDM to analyze water resources bearing capacity very well.

\section{Reference:}

[1]Haisheng, C., Prediction Research on Harbin Water Resources Carrying Capacity Based on A System Dynamic Model [D] , Harbin: Harbin Institute of Technology, 2014.6.

[2] Xiang, W., Jixiang, H., Lei, S., Jing, Z., Establishment of aquaculture water prediction model based on NAR neural network, Fishery Modernization, 4(42):30-34.2015.8.

[3] Information on http://www.stats.gov.cn/ 